\title{
Noninvasive fingerprinting-based tracking of replicative cellu- lar senescence using a colorimetric polyion complex array
}

\author{
Shunsuke Tomita, ${ }^{*} \dagger$ Hiroki Nomoto, ${ }^{\ddagger}$ Toru Yoshitomi, ${ }^{\ddagger}$ Kazutoshi Iijima, ${ }^{\S}$ Mineo Hashizume, ${ }^{\S}$ Keitaro \\ Yoshimoto ${ }^{*}, \dot{+}, / l$ \\ ${ }^{\dagger}$ Biomedical Research Institute, National Institute of Advanced Industrial Science and Technology, and DAILAB, 1-1-1 Hi- \\ gashi, Tsukuba, Ibaraki 305-8566, Japan. \\ * Department of Life Sciences, Graduate School of Arts and Sciences, The University of Tokyo, 3-8-1 Komaba, Meguro, \\ Tokyo 153-8902, Japan. \\ $\S$ Department of Industrial Chemistry, Faculty of Engineering, Tokyo University of Science, 12-1 Ichigayafunagawara-machi, \\ Shinjuku, Tokyo 162-0826, Japan. \\ " JST, PRESTO, The University of Tokyo, Komaba 3-8-1, Meguro, Tokyo 153-8902, Japan
}

\section{Supporting Information Placeholder}

\footnotetext{
1 ABSTRACT: A fingerprint-based sensing approach was used to 2 characterize in vitro cellular senescence. Secretion profiles of cul3 tured human fibroblasts in different senescent stages were trans4 formed into colorimetric enzyme-activity fingerprints by applying 5 cell-culture media to a polyion complex array. Analyzing the ob6 tained fingerprints using pattern recognition methods, such as lin7 ear discriminant analysis and hierarchical clustering analysis, re8 vealed that the polyion complex array allows the noninvasive track9 ing of the replicative senescence progress even in those stages 10 where a conventional marker such as senescence-associated $\beta$-ga11 lactosidase is negative. This fingerprint-based approach should 12 thus offer an effective way for the routine monitoring or screening 13 of in vitro cell-senescence studies.
}

14 Cellular senescence is an anti-proliferative program that limits 15 the propagation of cells subjected to different kinds of stimuli, such 16 as DNA damage, chromatin perturbations, activation of cancer 17 genes, and loss of telomeres after extensive proliferation. ${ }^{1}$ Alt18 hough cellular senescence has initially been considered as an irre19 versible cell-cycle-arrest mechanism that acts to protect against 20 cancer, recent discoveries have revealed that cellular senescence 21 may also be a detrimental biological process. For example, when 22 senescent cells are retained in tissue for prolonged periods of time, 23 these cells promote age-related tissue dysfunction by secreting fac24 tors that recruit local inflammation, induce aberrant tissue architec25 ture, and impair tissue homeostasis and regeneration. ${ }^{2,3}$

26 As the characterization of senescent cells in vivo has been limited 27 by difficulties associated with detecting and tracking senescent 28 cells in real tissue, most current insights regarding senescent cells 29 have been acquired using cell-culture experiments. ${ }^{3}$ Therefore, one 30 of the key issues in this field is the development of methods to ac31 curately characterize senescent cells in culture. For this purpose, a 32 variety of senescence-associated markers, ${ }^{1}$ especially senescence33 associated $\beta$-galactosidase (SA- $\beta-\mathrm{Gal}),{ }^{4-7}$ has been employed. 34 However, so far, the use of a combination of multiple markers is 35 still required, as a single universal marker for cell senescence has 36 not yet been discovered.
37 The secretory profile has attracted substantial attention in the 38 context of understanding and characterizing cultured senescent cell 39 populations, as cell senescence is accompanied by widespread 40 changes in the secretion of proteins, including inflammatory cyto41 kines, chemokines, growth factors, and proteases, which are known 42 as senescence-associated secretory phenotype (SASP) or senes43 cence-messaging secretome (SMS). ${ }^{3,8}$ Recent comprehensive pro44 tein analyses using antibody arrays have shown that senescence in45 ductions with a variety of stimuli have a common and significant 46 influence on secretion profiles for dozens of selected proteins. ${ }^{9-11}$

47 We have recently proposed a sensing strategy to acquire infor48 mation on secretory profiles in cell-culture media, and used it to 49 identify normal/cancer-cell types and stem-cell-differentiation lin50 eages. ${ }^{12}$ This strategy was developed using a fingerprint-based 51 sensing technique, which exploits the pattern recognition of unique 52 fingerprints for analytes. ${ }^{13}$ In general, fingerprints are generated 53 with arrays of cross-reactive molecules that can interact in different 54 ways with analytes. This technique has been employed for the de55 tection of proteins that are solubilized in dilute solutions ${ }^{14-18}$ or in 56 biological matrices. ${ }^{19-22}$ A distinct advantage of fingerprint-based 57 sensing is that it can also be applied to recognize the composition 58 of complex matrices themselves, such as serum ${ }^{21,23,24}$ and cell ly59 sate, ${ }^{15,25,26}$ even though not all components present may be 60 known. $^{27}$

61 Similarly, our fingerprint-based sensing of the secretory profile 62 was created without any information on the proteins secreted from 63 the cultured cells. ${ }^{12}$ In our approach, polyion complexes (PICs) be64 tween anionic enzymes and poly(ethylene glycol)-modified poly65 amines were used as cross-reactive molecules. ${ }^{21,28,29}$ The incuba66 tion of cell-culture media with the PICs causes the release of en67 zymes through competitive interactions between the secreted pro68 teins and the PICs, which generates unique fingerprints consisting 69 of the amount of changes in enzyme activity as variates. Inspired 70 by that work, we exploited a PIC array to recognize senescent-re71 lated secretomic profiles of normal human fibroblasts.

72 The current study is based on the hypothesis that the SASP pro73 duction due to replicative senescence could modulate the interac74 tion patterns between secreted proteins and individual PICs, which 


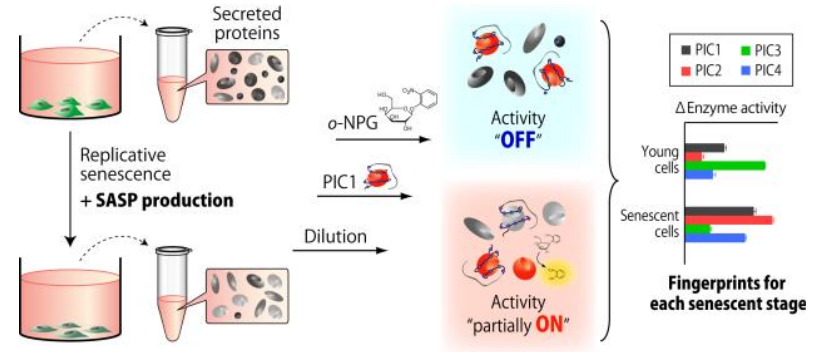

Figure 1. Schematic representation of the fingerprinting-based tracking of replicative senescence of human fibroblasts using a PIC array.

75 could generate enzyme-activity fingerprints that are unique to dif76 ferent senescent stages (Figure 1). In order to create PIC arrays that 77 are able to generate differential fingerprints for the tracking of se78 nescence progression, structurally diverse PICs are required. Based 79 on our previous report, ${ }^{12}$ we chose PICs with the following fea80 tures; (i) $\beta$-galactosidase from two different sources, i.e., from $\mathrm{As}$ 81 pergillus oryzae $\left(\mathrm{GAO}, M_{w}=110,000, p I=5.2\right)$ and Escherichia $82 \operatorname{coli}\left(\mathrm{GEC}, M_{w}=465,000, p I=5.1\right)$, and (ii) quaterinzed poly(eth83 ylene glycol)-block-poly( $N, N$-dimethylaminoethyl methacrylate) 84 (PEG- $b$-QPAMA) block co-polymers that contain functional 85 groups with different hydrophobicity, i.e., P1: benzyl groups (log $86 P=2.56)$ and P2: 1-hydroxyethyl groups $(\log P=-0.40)$ (Figure $872 \mathrm{~A})$. The $\beta$-galactosidase from either source is suitable for practical 88 use, owing to the high catalytic activity with respect to the hydrol89 ysis of chromogenic $o$-nitrophenyl- $\beta$-D-galactopyranoside ( $o$ NPG) 90 and the high structural stability. ${ }^{28,29}$

The addition of PEG- $b$-QPAMAs markedly decreased the cata92 lytic activity of both enzymes against $o$ NPG in $20 \mathrm{mM} 3-(N$-mor93 pholino)propanesulfonic acid (MOPS) buffer $(\mathrm{pH}=7.0$ ) (Figure 94 2B) due to the electrostatically driven formation of PIC. ${ }^{30,31}$ Con95 sistent with a previous report, ${ }^{29}$ hydrophobic P1 exerted a more 96 substantial effect on the inhibition of the enzymes.

97 Once the binding ratio that provided high inhibition had been 98 determined, we investigated the capability of the PIC array to track 99 cellular senescence. The conventional human fibroblast cell line 100 TIG-1 was chosen, considering its extensive use as a model cell 101 line for in vitro senescence studies. ${ }^{32,33}$ According to the literature, 102 replicative senescence was induced in TIG-1 by serial passag103 ing. ${ }^{32,33}$ The appearance of senescent cells was examined by the 104 SA- $\beta$-Gal assay, i.e., a histochemical detection of the $\beta$-galacto105 sidase activity. Although this assay may produce false-positive re106 sults under certain conditions such as confluence, it is the most 107 widely used method due to its general applicability and simplic108 ity. ${ }^{3,4}$ TIG-1 did not show a positive staining for conventional SA$109 \beta$-Gal assays until the increase in the population-doubling level $110(\triangle \mathrm{PDL})$ reached 56 (Figure $3 \mathrm{~A}$ ), i.e., the point where the cells ex111 hibited a markedly decelerated growth. Therefore, we examined the 112 tracking of the progress of TIG-1 senescence in the SA- $\beta$-Gal113 negative stages $(\triangle \mathrm{PDL}=6,19,34$, and 46$)$.

114 For the sample preparation, TIG-1 cells in different senescence 115 stages were seeded at $4 \times 10^{4}$ cells $/ \mathrm{cm}^{2}$ in Dulbecco's modified Ea116 gle's medium (DMEM) supplemented with $10 \%$ fetal bovine serum. 117 After 16 hours of incubation, the medium was replaced with a 118 chemically defined serum-free medium (CDCHO medium). The 119 culture supernatants were collected after 48 hours of incubation. 120 The obtained culture supernatants were diluted to a total protein 121 concentration of $5 \mu \mathrm{g} / \mathrm{mL}(20 \mu \mathrm{L})$ and then added to individual 122 wells of a 96-well plate, which contained solutions of PICs in 20
A
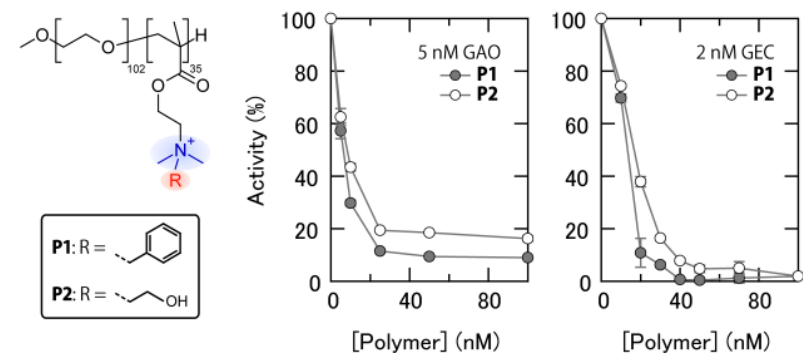

Figure 2. Changes in the enzyme activity upon addition of PEG$b$-QPAMAs. (A) Chemical structures of the PEG- $b$-QPAMA block co-polymers. (B) Normalized enzyme activity of $5 \mathrm{nM}$ GAO or $2 \mathrm{nM}$ GEC for $5 \mathrm{mM}$ oNPG with various concentrations of PEG- $b$-QPAMAs were measured in $20 \mathrm{mM}$ MOPS ( $\mathrm{pH}$ $=7.0$ ).

$123 \mathrm{mM}$ MOPS ( $\mathrm{pH}=7.0 ; 100 \mu \mathrm{L}$; for details, see the Supporting In124 formation). After $30 \mathrm{~min}$ of incubation, $25 \mathrm{mM} o \mathrm{NPG}$ in $20 \mathrm{mM}$ 125 MOPS $(\mathrm{pH}=7.0 ; 30 \mu \mathrm{L})$ was added, and the enzyme activity was 126 quantified using the time course of the absorbance at $400 \mathrm{~nm}$, gen127 erating a training matrix (4 PICs $\times 4 \Delta \mathrm{PDLs} \times 6$ replicates) (Table $128 \mathrm{~S} 1)$.

129 Figure 3B shows the changes in enzyme activity for four PICs. 130 The addition of culture supernatants resulted in increased enzyme 131 activities, indicating that competitive interactions occurred be132 tween PICs and secreted molecules, such as proteins. In order to 133 examine whether the individual fingerprints differ significantly, 134 these were subjected to a linear discriminant analysis (LDA), i.e., a 135 supervised pattern recognition method that produces a graphical 136 data output that provides insight into data clustering. ${ }^{13}$ In a linear 137 discriminant score plot (Figure 4A), each point represents the fin138 gerprint of a single analyte in the PIC array. The plot showed four 139 separated clusters corresponding to the individual $\triangle \mathrm{PDLs}$ in a 140 three-dimensional space. Interestingly, the cluster positions did not

A

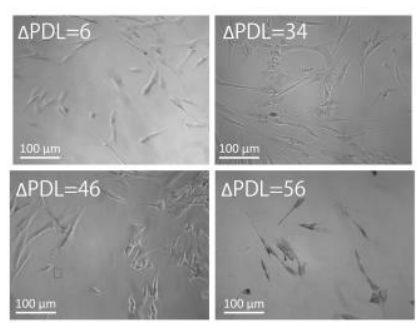

B

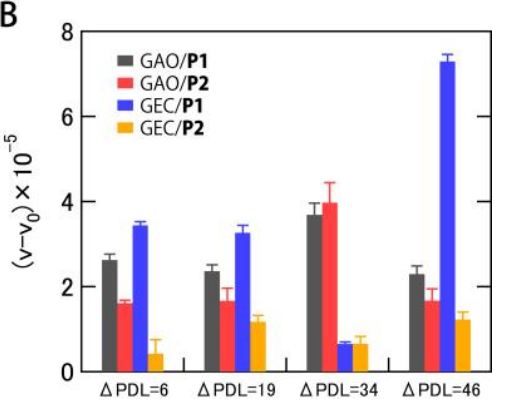

Figure 3. Fingerprinting of the secretomic profiles of TIG-1 in different senescent stages. (A) SA- $\beta$-Gal-staining of TIG-1. (B) Fingerprints of the changes in enzyme activity obtained from a PIC array. Values are shown as mean values $\pm \operatorname{SD}(n=6)$. 
141 change in a simple monotonous fashion with increasing $\triangle \mathrm{PDL}$, i.e., 142 in the initial senescence stages $(\triangle \mathrm{PDL}=6-19)$, the cluster moves 143 along the $z$-axis, which accounts for merely $0.4 \%$ of the total vari144 ance. After that, the cluster position varied markedly, especially on 145 the $x$ - (98.7\% of the total variance) and $y$-axes ( $0.9 \%$ of the total 146 variance). Thus, it seems feasible to assume that the selected PICs 147 are probably more susceptible to the changes in secretory profiles 148 regarding the later stages of TIG-1 senescence.

To evaluate the reliability of our sensing system to correctly 150 identify senescence levels, a cross-validation analysis was per151 formed. A leave-one-out procedure (jackknife classification analy152 sis) revealed accuracies of $46-83 \%$ when using one PIC, while 153 combinations of four PICs afforded $100 \%$ classification accuracy 154 (Table S2). Combined, our PIC array was able to track the gradual 155 senescence progress of TIG-1 by generating enzyme-activity fin156 gerprints.

157 In order to quantitatively investigate the relevance between the 158 response generation and the senescence progression, the distances 159 between all analytes were visualized via an unsupervised hierar160 chical clustering analysis (HCA) (Figure 4B), where the calculated 161 distance between analytes corresponds to similarities in the finger162 prints of the analytes. ${ }^{13}$ Again, four clusters corresponding to indi163 vidual values of $\triangle P D L$ were well distinguished in a horizontal den164 drogram, wherein $\triangle \mathrm{PDL}=19$ and $\triangle \mathrm{PDL}=46$ were initially clus165 tered, followed by $\triangle \mathrm{PDL}=6$ and $\triangle \mathrm{PDL}=34$. For TIG- 1 cells, 166 many factors such as telomere shortening, ${ }^{34}$ expression regulations 167 of genes including cell cycle inhibitors, ${ }^{35}$ mitochondria ${ }^{36}$ and other 168 proteins, ${ }^{35,37,38}$ are involved in cellular senescence. In general, 169 SASP is produced in a dynamic fashion during cellular senescence, 170 i.e., early SASP is dominated by the secretion of the transforming 171 growth factor- $\beta$ (TGF $\beta$ ) family, while subsequent signals of inter172 leukin- $1 \alpha \quad(\mathrm{IL}-1 \alpha)$ or $\mathrm{p} 38$-mitogen-activated protein kinase 173 (MAPK) cause late SASP, which consists of metalloproteinases, 174 IL-6, IL-8, and numerous other factors. ${ }^{3}$ Consequently, about half 175 of the analyzed 120 proteins exhibited significant differences with 176 respect to secretory quantities between presenescent and senescent 177 cells. ${ }^{9}$ Therefore, our time-course analysis implies that these com178 plicated and serial senescent-stage-dependent changes in the com179 position of proteins were sensed by the PIC array. Further investi180 gations of the correlation between response fingerprints of model 181 cells and gene/protein expression patterns at each senescent stage 182 should provide a more detailed understanding of not only the mech183 anism underlying the generation of fingerprints but that of cellular 184 senescence.

185 Finally, the similarities in the responses of individual PIC ele186 ments were analyzed to gain insight into the effective design of 187 cross-reactive PIC sets. A vertical dendrogram (Figure 4B) af188 forded two branches that comprise i) GAO-consisting PICs, and ii) 189 GEC-containing PICs. In other words, we observed a lower corre190 lation between the responses generated by PICs containing differ191 ent enzymes. Thus, it could be argued that the enzymes possibly 192 play a more dominant role in the interactions with secretomic com193 ponents related to senescence. Accordingly, the use of different en194 zymes may be effective to acquire diverse responses reflecting 195 TIG-1 senescence. The use of block co-polymers with different 196 functional groups that e.g. increase the hydrophobicity should lead 197 to a further diversification of responses and improve the discrimi198 nation ability.

199 In summary, we have developed a proof-of-concept study for the 200 fingerprinting-based tracking of replicative senescence of normal 201 human fibroblasts. Significantly, this sensing system is capable of 202 generating unique fingerprints that reflect different senescent levels, 203 even at the stage where the most commonly used senescent marker 204 SA- $\beta$-Gal is negative. In contrast to the conventional specific 205 marker-based methods, this fingerprint-based approach enables the 206 characterization of cultured cells based on the "overall" secretion
A
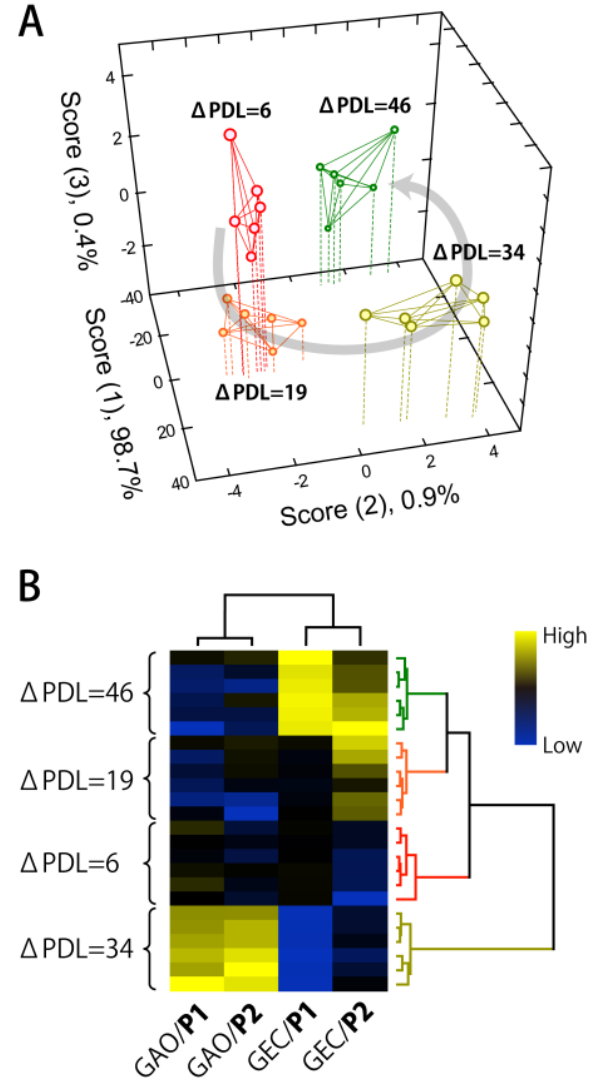

Figure 4. Analysis of the obtained fingerprints using pattern recognition methods. (A) Discriminant score plot for TIG-1 in four senescent stages obtained from the PIC array. The centers of the points that represent identical $\triangle \mathrm{PDL}$ (same color) are connected to facilitate a visual comparison between clusters. (B) Clustering analysis of the responses obtained using the PIC array. The hierarchical clustering dendrogram based on the Euclidean distance using the Ward method was created with a data set $(4$ PICs $\times 4 \Delta$ PDL $\times 6$ replicates). Therein, each element was standardized prior to the analysis based on the following equation; $z=(x-\mu) / \sigma$, wherein $z$ is the standardized score, $x$ the raw score, $\mu$ the mean of the population, and $\sigma$ the standard deviation of the population.

207 profiles in culture supernatants, which may contain rich infor208 mation on cell states. In this manner, accurate and noninvasive 209 sensing systems can be constructed without considering the 210 knowledge regarding any marker molecules. Given that various se211 nescent-inducing stimuli cause common influence on secretion pro212 files, ${ }^{9-11}$ we believe that the fingerprint-based approach could pro213 vide a versatile tool for the routine monitoring of cultured cells or 214 the screening of the influence of stimuli onto cell senescence.

215

\section{ASSOCIATED CONTENT}

\section{Supporting Information}

218 The Supporting Information is available free of charge on the ACS 219 Publications website.

220 Experimental details, fingerprint profiles, statistical analysis (Ta221 bles S1-S2).

222 


\section{${ }_{223}$ AUTHOR INFORMATION}

\section{${ }_{224}$ Corresponding Author}

$225 *$ E-mail: s.tomita@aist.go.jp.

226 *E-mail: ckeitaro@mail.ecc.u-tokyo.ac.jp

227 Notes

228 The authors declare the absence of any competing financial inter229 ests.

\section{${ }_{230}$ ACKNOWLEDGMENT}

231 This work was supported by JSPS KAKENHI grants 17H04884, 232 24350037, and 16K16398.

233

\section{${ }_{234}$ REFERENCES}

235 (1) Loaiza, N.; Demaria, M. Biochim. Biophys. Acta. 2016, 1865, 155-167. 236 (2) van Deursen, J.M. Nature 2014, 509, 439-446.

237 (3) Childs, B.G.; Gluscevic, M.; Baker, D.J.; Laberge, R.M.; Marquess, D.; 238 Dananberg, J.; van Deursen, J.M. Nat. Rev. Drug Discov. 2017, 16, 718239735.

240 (4) Debacq-Chainiaux, F.; Erusalimsky, J.D.; Campisi, J.; Toussaint, O. Nat. 241 Protoc. 2009, 4, 1798-1806.

242 (5) Lee, H.W.; Heo, C.H.; Sen, D.; Byun, H.O.; Kwak, I.H.; Yoon, G.; Kim, 243 H.M. Anal. Chem. 2014, 86, 10001-10005.

244 (6) Zhang, J.; Li, C.; Dutta, C.; Fang, M.; Zhang, S.; Tiwari, A.; Werner, 245 T.; Luo, F.T.; Liu, H. Anal. Chim. Acta. 2017, 968, 97-104.

246 (7) Lozano-Torres, B.; Galiana, I.; Rovira, M.; Garrido, E.; Chaib, S.; Ber247 nardos, A.; Munoz-Espin, D.; Serrano, M.; Martinez-Manez, R.; Sancenon, 248 F. J. Am. Chem. Soc. 2017, 139, 8808-8811.

249 (8) Kuilman, T.; Peeper, D.S. Nat. Rev. Cancer 2009, 9, 81-94.

250 (9) Coppe, J.P.; Patil, C.K.; Rodier, F.; Sun, Y.; Munoz, D.P.; Goldstein, J.; 251 Nelson, P.S.; Desprez, P.Y.; Campisi, J. PLoS Biol. 2008, 6, 2853-2868. 252 (10) Rodier, F.; Coppe, J.P.; Patil, C.K.; Hoeijmakers, W.A.; Munoz, D.P.; 253 Raza, S.R.; Freund, A.; Campeau, E.; Davalos, A.R.; Campisi, J. Nat. Cell 254 Biol. 2009, 11, 973-979.

255 (11) Yun, M.H.; Davaapil, H.; Brockes, J.P. eLIFE 2015, 4, e05505.

256 (12) Tomita, S.; Sakao, M.; Kurita, R.; Niwa, O.; Yoshimoto, K. Chem. Sci. 257 2015, 6, 5831-5836.

258 (13) Askim, J.R.; Mahmoudi, M.; Suslick, K.S. Chem. Soc. Rev. 2013, 42, 259 8649-8682.

260 (14) Chou, S.S.; De, M.; Luo, J.; Rotello, V.M.; Huang, J.; Dravid, V.P. J. 261 Am. Chem. Soc. 2012, 134, 16725-16733.

262 (15) Zamora-Olivares, D.; Kaoud, T.S.; Dalby, K.N.; Anslyn, E.V. J. Am. 263 Chem. Soc. 2013, 135, 14814-14820.

264 (16) Wang, M.; Ye, H.; You, L.; Chen, X. ACS Appl. Mater. Interfaces 2016, 3 $2658,574-581$.
266 (17) Tomita, S.; Ishihara, S.; Kurita, R. ACS Appl. Mater. Interfaces 2017, 267 9, 22970-22976.

268 (18) Tomita, S.; Matsuda, A.; Nishinami, S.; Kurita, R; Shiraki, K. Anal. 269 Chem. 2017, 89, 7818-7822.

270 (19) Miranda, O.R.; Chen, H.T.; You, C.C.; Mortenson, D.E.; Yang, X.C.; 271 Bunz, U.H.; Rotello, V.M. J. Am. Chem. Soc. 2010, 132, 5285-5289.

272 (20) Ran, X.; Pu, F.; Ren, J.; Qu, X. Chem. Commun. 2015, 51, 2675-2678. 273 (21) Tomita, S.; Niwa, O.; Kurita, R. Anal. Chem. 2016, 88, 9079-9086.

274 (22) Pode, Z.; Peri-Naor, R.; Georgeson, J.M.; Ilani, T.; Kiss, V.; Unger, 275 T.; Markus, B.; Barr, H.M.; Motiei, L.; Margulies, D. Nat. Nanotechnol. 276 2017, 12, 1161-1168.

277 (23) Xu, S.; Lu, X.; Yao, C.; Huang, F.; Jiang, H.; Hua, W.; Na, N.; Liu, 278 H.; Ouyang, J. Anal. Chem. 2014, 86, 11634-11639.

279 (24) Xu, S.; Wu, Y.; Sun, X.; Wang, Z.; Luo, X. J. Mater. Chem. B 2017, 280 5, 4207-4213.

281 (25) Rana, S.; Singla, A.K.; Bajaj, A.; Elci, S.G.; Miranda, O.R.; Mout, R.; 282 Yan, B.; Jirik, F.R.; Rotello, V.M. ACS Nano. 2012, 6, 8233-8240.

283 (26) Le, N.D.B.; Yesilbag Tonga, G.; Mout, R.; Kim, S.T.; Wille, M.E.; 284 Rana, S.; Dunphy, K.A.; Jerry, D.J.; Yazdani, M.; Ramanathan, R.; Rotello, 285 C.M.; Rotello, V.M. J. Am. Chem. Soc. 2017, 139, 8008-8012.

286 (27) Peveler, W.J.; Yazdani, M.; Rotello, V.M. ACS Sens. 2016, 1, 1282 2871285.

288 (28) Tomita, S.; Yoshimoto, K. Chem. Commun. 2013, 49, 10430-10432.

289 (29) Tomita, S.; Soejima, T.; Shiraki, K.; Yoshimoto, K. Analyst 2014, 139, 290 6100-6103.

291 (30) Kurinomaru, T.; Tomita, S.; Kudo, S.; Ganguli, S.; Nagasaki, Y.; Shi292 raki, K. Langmuir 2012, 28, 4334-4338.

293 (31) Kurinomaru, T.; Kuwada, K.; Tomita, S.; Kameda, T.; Shiraki, K. J. 294 Phys. Chem. B 2017, 121, 6785-6791.

295 (32) Wadhwa, R.; Ryu, J.; Gao, R.; Choi, I.K.; Morrow, G.; Kaur, K.; Kim, 296 I.; Kaul, S.C.; Yun, C.O.; Tanguay, R.M. J. Biol. Chem. 2010, 285, 3833 2973839.

298 (33) Udono, M.; Kadooka, K.; Yamashita, S.; Katakura, Y. Methods 2012, $29956,383-388$

300 (34) Takubo, K.; Aida, J.; Izumiyama, N.; Ishikawa, N.; Fujiwara, M.; Poon, 301 S.S.; Kondo, H.; Kammori, M.; Matsuura, M.; Sawabe, M.; Arai, T.; Baird, 302 D.M.; Nakamura, K. Mech. Ageing Dev. 2010, 131, 614-624.

303 (35) Udono, M.; Fujii, K.; Harada, G.; Tsuzuki, Y.; Kadooka, K.; Zhang, 304 P.; Fujii, H.; Amano, M.; Nishimura, S.; Tashiro, K.; Kuhara, S.; Katakura, 305 Y. Sci. Rep. 2015, 5, 17342.

306 (36) Kumazaki, T.; Sakano, T.; Yoshida, T.; Hamada, K.; Sumida, H.; Ter307 anishi, Y.; Nishiyama, M.; Mitsui, Y. Mech. Ageing Dev. 1998, 101, 91-99. 308 (37) Kumazaki, T.; Wadhwa, R.; Kaul, S.C.; Mitsui, Y. Exp. Gerontol. 1997, 309 32, 95-103.

310 (38) Inoue, C.; Zhao, C.; Tsuduki, Y.; Udono, M.; Wang, L.; Nomura, M.; 311 Katakura, Y. NPJ Aging Mech. Dis. 2017, 3, 11.

312

313

314 
Insert Table of Contents artwork here

Tracking the cellular senescence using a polyion complex array

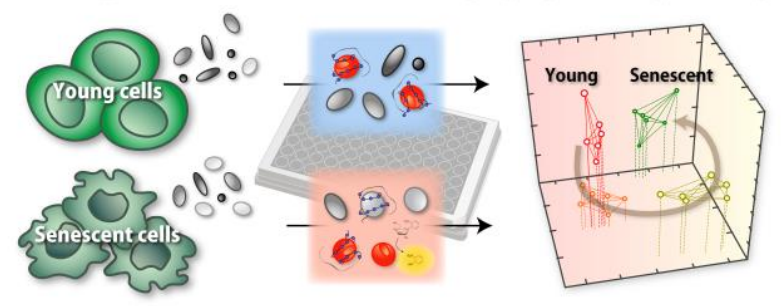

